\title{
Leaders
}

\section{Latest tests for prostatic neoplasia}

\author{
William J Marshall
}

Despite being the second most common cancer in males, and one from which mortality is increasing, cancer of the prostate remains in many ways an enigma. For example, little is known of its pathogenesis, although the androgenic hormones are clearly important, the incidence increases with age, and genetic factors also have a role. Furthermore, while its development appears to involve a progression from normal tissue through increasingly severe grades of dysplasia to intraepithelial neoplasia and thence to invasive cancer, there is considerable variation in the rates of progression to invasive disease. Indeed, previously unsuspected prostatic cancer is frequently found at necropsy ${ }^{1}$ and in men undergoing surgery for benign disease.

The diagnosis of prostatic cancer is histopathological and generally made from biopsy material. The decision to perform a biopsy may sometimes be based on clinical evidence alone, but for any cancer a diagnostic test to indicate its presence before it is clinically apparent should be of value (depending on the availability of effective treatment and the outcome if treatment is delayed). The screening tests available for prostatic cancer are digital rectal examination, transrectal ultrasound, and serum prostate specific antigen (PSA). Neither of the first two is sufficiently reliable on its own. In practice, measurements of PSA are used to decide whether to perform ultrasound examination as a preliminary to possible biopsy. However, PSA measurements can be used in several ways.

Unusually for a common tumour, there has long been a blood borne marker for prostatic cancer, namely prostatic acid phosphatase (PAP), first described in $1938 .^{2}$ However, PAP was not sufficiently sensitive or specific to be useful in screening, although it was of value in monitoring the response to treatment of patients with invasive disease. Prostate specific antigen (PSA) was identified in semen some 30 years later, and first isolated from prostatic tissue in $1979 .^{3}$ The first report of its potential as a tumour marker for prostatic cancer appeared shortly after ${ }^{4}$ and within a short time PSA had become the preferred marker and PAP became regarded as obsolete. Indeed, PAP has by now been deleted from most laboratories' repertoires.

But how reliable is PSA as a test for prostatic cancer? Are multiple measurements more reli- able than single measurements? Does the measurement of free PSA offer any advantage over that of bound PSA? And will PSA eventually go the way of PAP - are there any other tests for prostatic cancer in the offing? There are thousands of research papers on this topic and any review must necessarily be highly selective. Several more comprehensive reviews on screening for and managing prostatic cancer have been published recently. ${ }^{5-8}$

\section{Origin of PSA}

PSA is a serine protease secreted by cells lining the acini and ducts of the prostate. Its function is to hydrolyse the seminal fluid coagulum and assist in the liberation of spermatozoa. The small concentrations normally detectable in the blood are thought to reach it primarily by diffusion, passing from the ductal system across the prostatic stroma into capillaries and lymphatics. In prostatic disease, the anatomical disruption facilitates this process. The name PSA is actually misleading since the protein has been detected in various exocrine secretions; this is not of significance in men, but the measurement of PSA in breast secretions is being investigated as a possible screening test for carcinoma of the breast in women.

\section{Nature and isoforms}

PSA is a glycoprotein of molecular mass approximately $33 \mathrm{kDa}$. The native form is designated PSA-B; PSA-A is an active form with reduced carbohydrate content and PSA-C, PSA-D, and PSA-E have modified (internally cleaved) peptide chains and little enzymatic activity. Most PSA in seminal fluid is free, but in the blood, it binds extensively to the protease inhibitors $\alpha_{2}$ macroglobulin and $\alpha_{1}$ antichymotrypsin (ACT). ${ }^{9}$ This protein binding in plasma is a phenomenon common to all proteases and presumably is a protective mechanism. For PSA, it has considerable implications for the assay of the protein. When PSA is bound by $\alpha_{2}$ macroglobulin, it becomes encapsulated by the protein and its epitopes are hidden, with the result that it is not detected in immunoassays for PSA. Binding to ACT leaves most of the PSA epitopes exposed. Assays may therefore measure total (ACT bound + free) PSA, or either ACT bound or free PSA alone. The performance of the commercially available assays has recently been comprehensively reviewed. ${ }^{5}$ Early assays purported to measure total PSA 
but assays for free PSA are now widely available and assays which will measure free and total simultaneously are being developed.

\section{Reference intervals}

Although initially thought to be absent from the plasma when there is no prostatic disease, it is now clear that PSA is present in the plasma of healthy men, with a concentration range that is skewed to the right. PSA concentrations tend to increase with age, largely as a result of the increase in the size of the gland with age. ${ }^{10}$ Unfortunately, as with all other tumour markers, the concentrations of PSA found in healthy men and those found in the presence of cancer overlap: the concept of a "normal range" (strictly, "reference interval") in relation to PSA is thus inappropriate. Indeed, men may be healthy in that they do not have symptoms of prostatism and have a clinically or ultrasonographically normal prostate and yet still be harbouring cancer: the diagnosis can only be excluded on the basis of detailed necropsy examination. It is thus impossible to be certain that some members of a group used to define a reference interval may not have cancer. Furthermore, benign prostatic hypertrophy is a common condition so prostatic cancer may be present in a patient with benign disease and be contributing to the PSA present in the plasma.

PSA concentrations may also be increased in prostatitis and acute retention of urine. Digital rectal examination causes a small, transient increase (approximately 5\%, for up to 48 hours). ${ }^{11}$ The reported effects of recent ejaculation are inconsistent. There is no significant diurnal variation, but intraindividual variation can be more than $20 \%{ }^{12}$

When PSA measurement is used for screening or diagnosis (but not for monitoring known cancer), what is required is a measure of the probability that any given concentration can be associated with cancer. These can be used to define "action levels" to direct management. Ideally, the utility of these action levels should be audited with regard to their effect on outcome. The existence of different assays based on different antibodies and using different calibrators means that the results obtained using any one assay may not be strictly comparable with those obtained with another. In practice, with measurements of total PSA these differences probably matter little, and a plasma PSA concentration of up to $4 \mu \mathrm{g} /$ litre has usually taken as implying a very low risk of cancer (though not excluding it), while concentrations over $10 \mu \mathrm{g} /$ litre the probability of cancer becomes higher than that of benign disease. Men with concentrations at screening $>10$ $\mu \mathrm{g} /$ litre will usually be further investigated; those with concentrations below a lower cut off-usually taken as $4 \mu \mathrm{g} /$ litre, although values as low as $2.5 \mu \mathrm{g} /$ litre have been suggested-will not. It is in the concentration range of 4 to 10 $\mu \mathrm{g} /$ litre that uncertainty is greatest.

Improving the performance of PSA measurements in screening and diagnosis The ideal screening or diagnostic test would be positive in all individuals harbouring the disease in question (high sensitivity - that is, no false negatives) and negative in all unaffected individuals (high specificity-that is, no false positives). Given the overlap in the ranges of concentration of PSA that are seen in apparently healthy men and those with (histologically) proven cancer, PSA - in common with all other tumour markers - cannot be both $100 \%$ sensitive and $100 \%$ specific. Moving the cut off point or action limit to higher concentrations would increase specificity at the expense of sensitivity, while moving the cut off point lower has the opposite effect. ${ }^{13}$ Various strategies have been examined in attempt to improve the sensitivity of PSA measurements without compromising specificity.

\section{Age related cut off points}

Since plasma PSA concentration increases with age, the application of the same cut off in older men as in younger would have the effect of improving sensitivity but at the expense of specificity. Applying a higher cut off in older men would have the opposite effects. Age related cut off points have been proposed ${ }^{10}$ but are little used. Although a relatively lower cut off in younger men would detect more cancer and possibly be of greater long term benefit, more men would require further testing to establish the diagnosis. On the other hand, a relatively higher cut off in older men would result in decreased diagnosis of potentially curable (but not necessarily life threatening) tumours but less further testing -important not only for the individual but in economic terms, given the larger number of older men with raised PSA concentrations because of benign disease. These predictions were confirmed by the results of a study of over 4700 men. ${ }^{14}$ However, the prevalence of benign disease and cancer both increase with age, and the conclusions of a study of over 6600 men were that using a cut off for further investigation of $4 \mu \mathrm{g} /$ litre at all ages gives a similar biopsy rate and frequency of detection of cancer..$^{15}$ The arguments are the typical ones of screening - that is, the balancing of economic factors against possible clinical benefit, with the latter being uncertain on an individual basis.

\section{PSA "density"}

This concept relates serum PSA concentration to the size of the prostate as estimated using transrectal ultrasonography. There is some evidence that this technique increases sensitivity, particularly in the "grey" zone of total PSA $4-10 \mu \mathrm{g} /$ litre. ${ }^{16}{ }^{17}$ However, the choice of cut off is critical. Also, measurements of prostatic size by ultrasound have poor accuracy (magnetic resonance imaging is more accurate but not practical) and in practice the use of PSA density has not been shown to have a consistent advantage over the use of a simple cut off based on concentration alone. ${ }^{18}$

\section{PSA velocity}

In using cut off points to determine further management, a decision is being made about an individual based on observations on others. Repeating measurements of PSA in an 
individual allows comparison with his own previously determined values. PSA concentration increases linearly with time (at about 0.12 $\mu \mathrm{g} /$ litre/year) as a result of benign hypertrophy; the rate is higher and the increase exponential in cancer and relatively higher with more aggressive tumours. ${ }^{19}$ Either an increase in velocity or a high velocity ( $>20 \%$ annual increase $)^{20}$ is thus suggestive of cancer. Problems with this technique include the difficulty of ensuring comparability of measurements made over a period of years (requiring a very stable assay system) and the innate intraindividual variation of PSA measurements. ${ }^{12}$ This latter means that an apparent increase in PSA from 4.0 to $5.2 \mu \mathrm{g} /$ litre has a probability of less than 0.95 of being a true increase. Ideally, PSA velocities should be based on sets of several measurements in order to reduce the effect of this variation. PSA velocity has no better sensitivity than simple cut offs but has better specificity. Used alone, however, it might delay diagnosis, since a rise can only be discerned in retrospect. On the other hand, its use should allow earlier detection of cancers since an increase in PSA might be significant even if the absolute value remains below $4 \mu \mathrm{g} /$ litre. Whether this would have any effect on outcome is another matter.

\section{Free PSA and free/bound PSA ratio}

Total, bound, and free PSA concentrations increase in parallel with age, so that the ratio of free to bound remains constant. ${ }^{21}$ The most promising advances in the use of PSA in the diagnosis of prostatic cancer stem from the discovery that the proportion of protein bound PSA in the plasma is higher in cancer than in benign disease ${ }^{22}$ (there is evidence that ACT synthesis often occurs in neoplastic prostatic tissue but only rarely in benign tissue). ${ }^{23}$ Although there have been methodological problems with the free PSA assays, the use of ratios does promise enhanced sensitivity and specificity, albeit at the cost of doing two measurements and the increased imprecision that the use of a ratio of two concentrations implies.

Ratios would not help in individuals found to have high total PSA ( $>10 \mu \mathrm{g} /$ litre) since they would always require further investigation, and-except possibly in young men-would be of little value with low total concentrations. The greatest benefit would be in the intermediate group, for example men with a total PSA of between 4 and $10 \mu \mathrm{g} /$ litre, where the finding of a high free/total ratio might spare men a biopsy and allow a more conservative approach (for example, annual repeat PSA measurements and digital rectal examination), while a low ratio would be an indication for transrectal ultrasound examination and biopsy. The results of several studies have confirmed this prediction. $^{2425}$

This topic is presently the subject of intensive research. ${ }^{26}$ It appears that measurement of the free/bound PSA ratio is a significant improvement over the measurement total PSA alone for the diagnosis of early prostatic cancer.
Relating the free/bound PSA ratio to prostate volume may help further. ${ }^{27}$ While it must follow that if fewer biopsies are performed some cancers will be missed, however many might have proven negative, it is also important to avoid unnecessary biopsies and operations.

\section{Are there alternatives to PSA?}

Several substances other than PSA have been detected at increased concentration in the plasma of patients with prostatic cancer (for example, creatine kinase BB, $\alpha$-fetoprotein, and carcinoembyronic antigen). None seems likely to supplant PSA. What can be predicted is that assays for PSA will continue to be improved as manufacturers compete in an increasingly informed market place; that research into the best way to apply PSA measurements will continue; and that as data from individual groups continue to be published, systematic reviews will be performed which may further help to define the role of PSA.

\section{Conclusion}

At present, there is no convincing evidence of consistent clinical benefit from the early detection of prostatic cancer ${ }^{28}$ although this is not equivalent to there being evidence of no benefit. ${ }^{29}$ Trials are currently under way but will not be completed until the early years of the next century. ${ }^{3031}$ The advent of PSA has raised the question of whether, since prostatic cancer is so common, asymptomatic men should be routinely screened by PSA measurement. In the USA, doing this has led to a considerable increase in the number of men having biopsies and prostatectomies. Advice in the United Kingdom is that routine screening is not appropriate ${ }^{6}$ but this may not stop patients requesting it. ${ }^{32}$ Laboratories can anticipate increasing numbers of requests for PSA and should modify their practice with regard to the precise choice of technique according to the evidence.

I thank Dr WS Wassif and the anonymous reviewer for their helpful comments on the manuscript.

1 Franks LM. Latent carcinoma of the prostate. F Pathol Bacteriol 1954;68:603-16.

2 Gutman AB, Gutman EB. An "acid" phosphatase occurring in the serum of patients with metastasizing carcinoma of the prostate gland. $\mathcal{F}$ Clin Invest 1938;17:473-8.

3 Wang MC, Valenzuela LA, Murphy GP, et al. Purification of a human prostate specific antigen. Invest Urol 1979;17:15963.

4 Papsidero LD, Wang MC, Valenzuela LA, et al. A prostate antigen in the sera of prostatic cancer patients. Cancer Res antigen in the sera

5 Sikaris KA. Prostate specific antigen. Clin Biochem Revs 1996;17:50-68.

6 Chamberlain J, Melia J, Moss S, et al. Report prepared for the health technology assessment panel of the NHS executive on the diagnosis, management, treatment and costs of prostate cancer in England and Wales. $\mathrm{Br} \quad \mathcal{F}$ Urol 1997;79 (suppl 3): 1-32.

7 Freid RM, Davis NS, Weiss GH. Prostate cancer screening and management. Med Clin North Am 1997;81:801-22.

8 Frydenberg M, Stricker PD, Kaye KW. Prostate cance diagnosis and management. Lancet 1997;349:1681-6.

9 Lilja H, Christensson A, Dahlen U, et al. Prostate-specific antigen in serum occurs predominantly in complex with alpha-1-antichymotrypsin. Clin Chem 1991;37:1618-25.

10 Oesterling JE, Jacobsen SJ, Chute CG, et al. Serum prostate specific antigen in a community-based population of healthy men: establishment of age specific reference ranges. $\mathcal{F} A M A$ 1993;270:860-4. 
11 Thomson RD, Clejan S. Digital rectal examinationassociated alterations in serum prostate-specific antigen. associated alterations in serum pros

12 Prestigiacomo AF, Stamey TA. Physiological variation of serum prostate specific antigen in the 4 to $10 \mathrm{ng} / \mathrm{mL}$ range in male volunteers. F Urol 1996;155:1977-80.

13 Marshall WJ. The interpretation of laboratory data. In Marshall WJ, Bangert SK, eds. Clinical biochemistry. Edinburgh: Churchill Livingstone, 1995:15-24.

14 El-Galley RES, Petros JA, Sanders WH, et al. Normal range prostate-specific antigen in screening prostate adenocarcinoma. Urology 1995;46:200-4.

15 Catalona WJ, Hudson MA, Scardino PT, et al. Selection of optimum prostate specific antigen cutoffs for early detection of prostate cancer: receiver operating characteristics curves. F Urol 1994;152:2037-42.

16 Nishiya M, Miller GJ, Lookner DH, et al. Prostate specific antigen density in patients with histologically proven prosatigen density in patients with histologicer

17 Mettlin C, Littrup PJ, Kane RA, et al. Relative sensitivity and specificity of serum prostate specific antigen (PSA) and specificity of serum prostate specific antigen (PSA) PSA change. Cancer 1994; 74:1615-20.

18 Catalona WJ, Richie JP, deKernion JB, et al. Comparison of prostate specific antigen concentration versus prostate specific antigen density in the early detection of prostate cancer: receiver operating characteristics curves. F Urol 1994;152:2031-6.

19 Carter HB, Pearson JD, Metter J, et al. Longitudinal evaluation of prostate-specific antigen levels in men with and without prostate disease. $\mathcal{F A M A}$ 1992;267:2215-20.

20 Brawer MK, Beatie J, Wener MH, et al. Screening for prostatic carcinoma with prostate specific antigen: results of second year. F Urol 1993;150:106-9.

21 Oesterling JE, Jacobsen SJ, Klee GG, et al. Free, complexed and total serum prostate specific antigen: the establishment of appropriate reference ranges for their concentrations and ratios. F Urol 1995;154:1090-5.

22 Stenman UH, Leinonen J, Alfthan N, et al. A complex between prostate-specific antigen and alpha-1- antichymotrypsin is the major form of prostate-specific antigen in serum of patients with prostate cancer: assay of the complex improves clinical sensitivity for cancer. Cancer Res 1991;51:222- 6 .

23 Bjork T, Bjartell A, Abrahamsson PA, et al. Alpha 1-antichymotrypsin production in PSA-producing cells is common in prostate cancer but rare in benign prostatic hyperplasia. Urology 1994;43:427-34.

24 Catalona WJ, Smith DS, Wolfert RL, et al. Evaluation of percentage of free serum prostate-specific antigen to improve specificity of prostate cancer screening. $\mathcal{F} A M A$ 1995;274:1214-20.

25 Demura $\mathrm{T}$, Shinohara N, Tanaka $\mathrm{M}$, et al. The ratio of free to total serum prostate specific antigen: a method of detecting prostate carcinoma. Cancer 1996;77:1137-43.

26 Moore A. Free PSA as a percentage of the total: where do we go from here? Clin Chem 1997;43:1561-2.

27 Stephan C, Lein M, Jung K, et al. The influence of prostate volume on the ratio of free to total prostate specific antigen in serum of patients with prostate carcinoma and benign prostatic hyperplasia. Cancer 1997;79:104-9.

28 Woolf SH. Screening for prostate cancer with prostatespecific antigen: an examination of the evidence. $N$ Engl f Med 1995;333:1401-5.

29 The prostate question, unanswered still. Lancet 1997;349: 443.

30 Gohogan JK, Prorok PC, Kramer BS, et al. Prostate cancer screening in the prostate, lung, colorectal and ovarian cancer screening trial of the National Cancer Institute. 7 Urol 1994;152:1905-9.

31 Wilt TJ, Brawer MK. The Prostate Cancer Intervention Versus Observation Trial: a randomised trial comparing radical prostatectomy versus expectant management for the treatment of clinically localised prostate cancer. 7 Urol 1994;152:883-4.

32 Woolf $\mathrm{SH}$. Should we screen for prostate cancer? $\mathrm{BMF}$ 1997;314:989-90. 